\title{
Papers
}

\section{Cognitive behaviour therapy for adolescents with chronic fatigue syndrome: randomised controlled trial}

\author{
Maja Stulemeijer, Lieke W A M de Jong, Theo J W Fiselier, Sigrid W B Hoogveld, Gijs Bleijenberg
}

\begin{abstract}
Objective To evaluate the efficacy of cognitive behaviour therapy for adolescents aged 10-17 years with chronic fatigue syndrome.

Design Randomised controlled trial.

Setting Department of child psychology.

Participants 71 consecutively referred patients with chronic fatigue syndrome; 36 were randomly assigned to immediate cognitive behaviour therapy and 35 to the waiting list for therapy.

Intervention 10 sessions of therapy over five months. Treatment protocols depended on the type of activity pattern (relatively active or passive). All participants were assessed again after five months.

Main outcome measures Fatigue severity (checklist individual strength), functional impairment (SF-36 physical functioning), and school attendance.

Results 62 patients had complete data at five months (29 in the immediate therapy group and 33 on the waiting list). Patients in the therapy group reported significantly greater decrease in fatigue severity (difference in decrease on checklist individual strength was $14.5,95 \%$ confidence interval 7.4 to 21.6 ) and functional impairment (difference in increase on SF-36 physical functioning was 17.3, 6.2 to 28.4) and their attendance at school increased significantly (difference in increase in percentage school attendance was 18.2, 0.8 to 35.5). They also reported a significant reduction in several accompanying symptoms. Self reported improvement was largest in the therapy group. Conclusion Cognitive behaviour therapy is an effective treatment for chronic fatigue syndrome in adolescents.
\end{abstract}

\section{Introduction}

Patients with chronic fatigue syndrome have debilitating unexplained severe fatigue that is not the result of an organic disease or ongoing exertion and is not alleviated by rest. Symptoms last for at least six months and are accompanied by other symptoms like muscle pain and unrefreshing sleep. ${ }^{12}$ This condition can occur in adults and adolescents. ${ }^{3}$

Several randomised controlled trials have shown that cognitive behaviour therapy is effective in adults. ${ }^{45}$ To date, however, there have been no published controlled studies on such therapy for adolescents, though one uncontrolled study suggested that such a behavioural approach can reduce fatigue in adolescents. ${ }^{6}$ Development of potentially effective interventions is especially important in young people to avoid prolonged absence from school and restricted social activities, which threaten healthy development. ${ }^{7-9}$

\section{Methods}

We studied the efficacy of cognitive behaviour therapy for adolescents with chronic fatigue syndrome by comparing outcome in those randomly assigned to immediate therapy with outcome in those who were assigned to the waiting list for therapy. We used two treatment protocols: one for patients with a passive physical activity pattern and one for relatively active patients. ${ }^{4} 1011$

We hypothesised that fatigue severity, functional impairment, and school absence would decrease significantly more in those assigned to immediate therapy.

\section{Patients}

As part of the usual care all consecutive patients with a major complaint of fatigue referred to the paediatrics outpatient clinic between October 1999 and October 2002 were assessed by means of a detailed history and physical and laboratory examinations. Patients were eligible if they were between 10 and 17.2 years of age (to allow the older participants to complete therapy before their 18th birthday) and met the US Centres for Disease Control Prevention criteria for chronic fatigue syndrome. ${ }^{1}$ Severe fatigue and severe functional impairment were defined as a score of 40 or more on the fatigue severity subscale of the checklist individual strength ${ }^{4}$ and a weighted score of 65 or less on the SF-36 physical functioning subscale. We excluded patients with psychiatric comorbidity, as assessed during an interview with both patients and parents by an experienced child psychologist before randomisation.

\section{Design and procedures}

We gave patients and their parents verbal and written information about the study and obtained informed consent before randomisation. Before baseline assessments, patients were randomly allocated to one of the two groups by means of a sequence of labelled cards contained in sealed numbered envelopes that were prepared by a statistical adviser and opened by the researcher in the presence of patient and parent(s). The randomisation sequence was based on a table of random numbers. The same researcher performed the allocation procedure and data analyses.

Patients assigned to immediate therapy had to agree to not having any further medical examinations or other treatments for fatigue during therapy. ${ }^{4}$ Patients assigned to the waiting list were assessed directly after randomisation and five months later. No further requirements were made during the waiting period, and they were free to have other examinations or treatments. They were informed beforehand that, if desired, they could start therapy directly after the second assessment. 


\section{Intervention}

The therapy comprised 10 individual sessions over five months. We used two treatment protocols based on the existing protocols for adults and adapted for the two types of patterns of physical activity. ${ }^{41}$ Adolescents with a relatively active physical activity pattern alternate between periods of activity and periods of rest. ${ }^{11}$ In contrast, those with a passive physical activity pattern spend most time lying down and go out infrequently. Most do not attend school at all.

Active patients-For relatively active patients treatment started with them learning to recognise and accept their current state of fatigue and impairment. Subsequently, they reduced their levels of activity and learnt to respect the limitations. After achieving this balance, the patient started to build up activity levels. This protocol was used in the trial of Prins et al.

Passive patients-For passive patients we started a systematic programme of activity building as soon as possible. To assure adherence, we first addressed and challenged their beliefs that activity would aggravate symptoms. In such patients it is thought to be counterproductive to reduce activity levels any further or reinforce the patient's need to respect limitations. ${ }^{11}$

All therapy-Both protocols differed from the treatment of adults. Firstly, we actively involved parents in supporting their child and explored and addressed parents' beliefs and behaviours regarding the condition of their child. Secondly, the aims of therapy took into account the specific developmental tasks of adolescents. In children younger than 15 years, parents often acted as a coach; for older participants, parents had to step back and encourage their child to take responsibility for the treatment. Thirdly, return to full time education was always a goal of treatment, and a plan for returning to school was discussed early with everyone involved. Four child therapists who were trained and supervised by an experienced cognitive behavioural therapist administered all therapy.

\section{Primary outcome variables}

We measured fatigue with the fatigue severity subscale of the checklist individual strength, a questionnaire originally developed for adults. ${ }^{42}{ }^{13}$ Internal consistency was excellent, with a Chronbach's $\alpha$ of 0.93 . We measured functional impairment with the "physical functioning" subscale of the SF-36 (range 0 (maximum physical limitation) to 100 (ability to do vigorous activity)). This measure is reliable and valid and has been used in adolescents with chronic fatigue syndrome. ${ }^{14}{ }^{15}$ School attendance was calculated by dividing the hours that the patient attended lessons in the previous week by the hours that the patient should have attended.

\section{Other outcome variables}

We rated the presence of nine symptoms (see table 3 below) on a 4 point Likert scale (from never to every day). ${ }^{1}$ Patients indicated whether they were completely recovered, felt much better, had the same complaints, or had become worse than at the previous assessment. ${ }^{46}$ We measured physical activity with an actometer, a motion sensing device attached to the ankle and worn continuously for 12 days. $^{10}$ Patients were classified by comparison of daily activity scores with the reference score of adults with chronic fatigue syndrome. Pervasively passive patients had an average daily activity score below this reference score on 11 or 12 of a total of 12 days. All other patients were considered to be relatively active. ${ }^{4} 1017$

\section{Analysis}

Power calculations showed that we needed 30 patients in each group to achieve $90 \%$ power to detect a difference of 7 points on the fatigue severity subscale with an $\alpha<5 \%$ (two tailed). ${ }^{18}$ We used SPSS (version 10.0) for all statistical analyses. Effects were tested at the $\mathrm{P}<0.05$.

We analysed data on an intention to treat basis and carried forward last observations in cases of missing data. Differences between groups on the amount of change in the primary outcome variables were calculated with analyses of variance on differences in scores before and after the five months, with 95\% confidence intervals. We defined patients as having clinically significant improvement if they had a reliable change index $>1.96$ and a fatigue score $<35.7$ ( $1 \mathrm{SD}$ above the mean for 420 healthy adolescents, unpublished data) for fatigue severity ${ }^{19}$; had an increase of $\geq 50$ or an end score of $\geq 75$ for physical functioning ${ }^{20}$; were fully attending school at the five months assessment; and reported "I have completely recovered" or "I feel much better." We used $\chi^{2}$ tests to assess differences between the proportions of patients meeting these criteria.

\section{Results}

The figure shows the trial profile. Seventy one patients were randomly allocated to either immediate therapy $(n=36)$ or to remain on the waiting list $(n=35)$. After randomisation we excluded two patients (one from each group) because the diagnosis of chronic fatigue syndrome was incorrect. Analyses were based on the 69 remaining patients. Of those, 29 in the immediate therapy group and 33 from the waiting list completed the assessment at five months. Six patients dropped out during the course of treatment, three of them did not finish the second assessment. Table 1 shows the baseline characteristics of both groups.

\section{Effect of intervention}

Primary outcome-Patients in the immediate therapy group reported a significantly greater decrease in fatigue severity (difference in decrease on checklist individual strength 14.5, 95\% confidence interval 7.4 to 21.6) and functional impairment (difference in increase on SF-36 physical functioning 17.3, 6.2 to 28.4) than patients on the waiting list. School attendance also increased significantly more in the therapy group (difference in increase in school attendance 18.2, 0.8 to 35.5) (table 2).

Other outcomes-At five months the participants in the therapy group reported a significantly greater decrease in how often they felt ill after exercise, impaired concentration, unrefreshing sleep, muscle pain, and headache (table 3). Patients on the waiting list reported increased prevalence of impaired concentration, unrefreshing sleep, and muscle pain.

Clinically significant improvement-For all primary outcome variables as well as for self rated improvement the proportion of patients with clinically significant improvements was greatest among those in the therapy group (table 4).

Two treatment protocols-There were no significant differences in all primary outcomes between adolescents who were treated with the protocol designed for patients with a passive physical activity pattern and those who were treated with the protocol for more active patients (table 5).

\section{Discussion}

In adolescents with chronic fatigue, cognitive behaviour therapy was more effective than remaining on a waiting list in reducing severity of fatigue, improving physical functioning, and increasing school attendance. Furthermore, treatment resulted in a greater proportion of patients with clinically significant change in these variables, more self reported improvement, and 


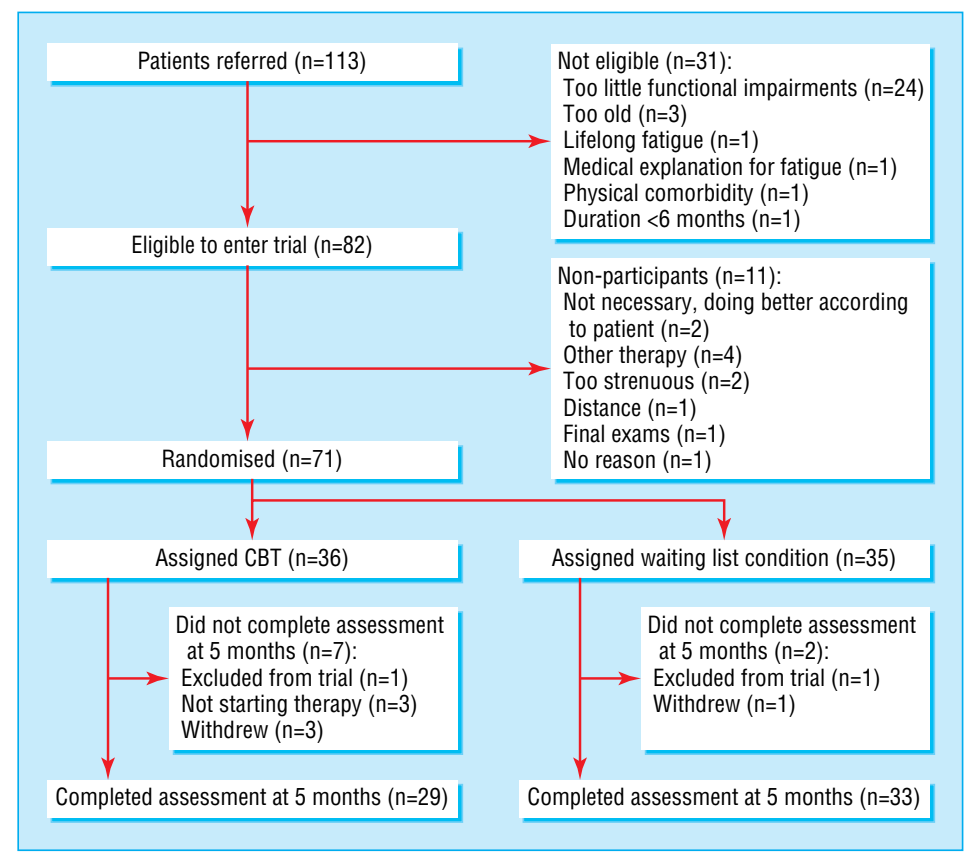

Trial profile

a significant reduction in several additional symptoms. These results endorse the findings of previous studies on the efficacy of cognitive behaviour therapy for adults with chronic fatigue syn-

Table 1 Baseline characteristics of study participants. Values are means (SD) unless stated otherwise

\begin{tabular}{|c|c|c|}
\hline & $\begin{array}{l}\text { Cognitive behaviour therapy } \\
\qquad(\mathrm{n}=35)\end{array}$ & Waiting list $(n=34)$ \\
\hline \multicolumn{3}{|l|}{ Demography } \\
\hline Age (years) & $15.6(1.3)$ & $15.7(1.3)$ \\
\hline $\begin{array}{l}\text { Median duration of complaints } \\
\text { (months) }\end{array}$ & 16.0 & 18.0 \\
\hline No (\%) female & $31 / 35(89)$ & $31 / 34(91)$ \\
\hline \multicolumn{3}{|l|}{ Primary outcome variables } \\
\hline $\begin{array}{l}\text { Fatigue severity (checklist } \\
\text { individual strength) }\end{array}$ & $52.5(3.8)$ & $51.6(4.3)$ \\
\hline Physical functioning (SF-36) & $42.1(16.5)$ & $45.3(17.0)$ \\
\hline \multicolumn{3}{|l|}{ No (\%) with school attendance: } \\
\hline Full & $4 / 35(11)$ & $6 / 34(18)$ \\
\hline Partial & $31 / 35$ (89) & $28 / 34(82)$ \\
\hline \multicolumn{3}{|l|}{ Physical activity pattern (№ (\%)) } \\
\hline Pervasively passive & $10 / 33^{*}(30)$ & $7 / 34 \quad(21)$ \\
\hline Relatively active & $23 / 33^{*}(70)$ & $27 / 34(79)$ \\
\hline
\end{tabular}

*Two participants refused to wear an actometer. drome. ${ }^{45}$ Passive and active patients showed equal improvements on all primary outcome variables. Furthermore, rates of improvement were larger than seen in the study by Prins et al, in which only one protocol was used to treat all patients. ${ }^{4}$

We tried to maximise inclusion by repeatedly informing general practitioners and paediatricians about the study and prolonging recruitment. Nevertheless, our final samples were still relatively small. This may be due to underdiagnosis because of unfamiliarity with adolescent chronic fatigue syndrome or may point to reluctance in doctors to diagnose this syndrome. Alternatively, chronic fatigue syndrome may be less common than previously estimated. ${ }^{3}$ We believe that our results can be generalised to other adolescents who fulfil the diagnostic criteria for chronic fatigue syndrome as our patients were referred from a large part of the Netherlands.

Six patients (19\%) withdrew from therapy, which is higher than in most adult studies on cognitive behaviour therapy in patients with chronic fatigue syndrome. ${ }^{5}$ Most withdrawals occurred in the first half of the study, suggesting that therapists became more experienced in meeting the specific need for enhancing motivation of adolescent patients. As we did not have reference scores for activity pattern in adolescents, we used scores for adults. Fortunately, our results showed that mean

Table 2 Effect of cognitive behaviour therapy on fatigue severity, functional impairment, and school attendance

\begin{tabular}{|c|c|c|c|c|}
\hline \multirow[b]{2}{*}{ Condition* ${ }^{*}$} & \multicolumn{2}{|c|}{ Mean score (SD) } & \multirow[b]{2}{*}{ Treatment effect† $(95 \% \mathrm{CI})$} & \multirow[b]{2}{*}{$P$ value } \\
\hline & O months & 5 months & & \\
\hline \multicolumn{5}{|l|}{ Fatigue severity } \\
\hline Cognitive behaviour & $52.5(3.8)$ & $30.2(16.8)$ & 14.5 (7.4 to 21.6$)$ & 0.001 \\
\hline Waiting list & $51.6(4.4)$ & $44.0(13.4)$ & & \\
\hline \multicolumn{5}{|l|}{ Physical functioning } \\
\hline Cognitive behaviour & $42.1(16.5)$ & $69.4(28.0)$ & 17.3 (6.2 to 28.4) & 0.003 \\
\hline Waiting list & $45.3(17.0)$ & $55.3(21.1)$ & & \\
\hline \multicolumn{5}{|l|}{ School attendance } \\
\hline Cognitive behaviourł & $46.2(38.9)$ & $74.7(37.8)$ & 18.2 (0.8 to 35.5$)$ & 0.040 \\
\hline Waiting list & $56.4(38.6)$ & $66.7(36.0)$ & & \\
\hline
\end{tabular}

${ }^{*}$ Cognitive behaviour therapy $n=35$; waiting list $n=34$.

†Difference in improvement between intervention and control group.

$\ddagger$ Two participants were left out of analyses because they had taken their final examinations and therefore were not required to attend school at five months. 
Table 3 Effects of treatment on the prevalence of additional symptoms according to the Centers of Disease Control criteria. Values are means (SD)

\begin{tabular}{|c|c|c|c|c|}
\hline \multirow[b]{2}{*}{ Condition* } & \multicolumn{2}{|c|}{ Symptom score ${ }^{\dagger}$} & \multirow[b]{2}{*}{ Treatment effect (95\% CI) } & \multirow[b]{2}{*}{$P$ value } \\
\hline & 0 months & 5 months & & \\
\hline \multicolumn{5}{|l|}{ Unrefreshing sleep } \\
\hline Cognitive behaviour & $3.4(1.0)$ & $2.5(1.1)$ & $-1.2(-1.8$ to -0.6$)$ & 0.001 \\
\hline Waiting list & $2.7(0.6)$ & $3.2(0.8)$ & & \\
\hline \multicolumn{5}{|l|}{ Muscle pain } \\
\hline Cognitive behaviour & $2.7(1.1)$ & $2.4(1.0)$ & $-1.1(-1.6$ to -0.6$)$ & 0.001 \\
\hline Waiting list & $1.8(0.9)$ & $2.7(0.8)$ & & \\
\hline \multicolumn{5}{|c|}{ Impaired concentration } \\
\hline Cognitive behaviour & $2.9(1.1)$ & $2.4(1.2)$ & $-1.1(-1.5$ to -0.65$)$ & 0.001 \\
\hline Waiting list & $2.0(0.9)$ & $2.7(0.8)$ & & \\
\hline \multicolumn{5}{|c|}{ Tiredness after exercise } \\
\hline Cognitive behaviour & $3.5(0.7)$ & $2.5(1.1)$ & $-1.0(-1.5$ to -0.5$)$ & 0.001 \\
\hline Waiting list & $2.8(0.3)$ & $2.9(0.3)$ & & \\
\hline \multicolumn{5}{|l|}{ Headache } \\
\hline Cognitive behaviour & $2.9(0.8)$ & $2.6(0.9)$ & $-0.05(-0.9$ to 0.0$)$ & 0.033 \\
\hline Waiting list & $2.3(0.9)$ & $2.5(0.8)$ & & \\
\hline \multicolumn{5}{|l|}{ Impaired memory } \\
\hline Cognitive behaviour & $2.1(1.2)$ & $1.8(1.1)$ & $-0.4(-0.93$ to 0.1$)$ & 0.12 \\
\hline Waiting list & $2.2(1.0)$ & $2.4(1.0)$ & & \\
\hline \multicolumn{5}{|l|}{ Multi-joint pain } \\
\hline Cognitive behaviour & $2.5(1.2)$ & $2.0(1.2)$ & $-0.2(-0.7$ to 0.3$)$ & 0.38 \\
\hline Waiting list & $2.6(0.6)$ & $2.3(0.9)$ & & \\
\hline \multicolumn{5}{|l|}{ Sore throat } \\
\hline Cognitive behaviour & $1.8(0.8)$ & $1.6(0.8)$ & $0.2(-0.3$ to -0.7$)$ & 0.40 \\
\hline Waiting list & $2.3(0.8)$ & $1.9(0.7)$ & & \\
\hline \multicolumn{5}{|c|}{ Sensitive lymph nodes } \\
\hline Cognitive behaviour & $1.8(1.0)$ & $1.6(0.9)$ & $0.0(-0.4$ to 0.6$)$ & 0.72 \\
\hline$\overline{\text { Waiting list }}$ & $1.8(0.9)$ & $1.5(0.9)$ & & \\
\hline
\end{tabular}

*Cognitive behaviour therapy $n=35$; waiting list $n=34$.

†Symptoms rated on 4 point Likert scale (never to every day), range 1 to 4 .

activity levels and distributions of types of activity were similar to those in adults. Thus the use of reference scores for adults should not have led to misclassification.

Almost $60 \%$ of the patients in the immediate therapy group returned to full time education, an important indication of recovery. We consider that return to school is most difficult when adolescents lose contact with school and classmates and experience longstanding social isolation. In these cases other possibilities to guarantee education should be explored, such as considering a new school.

Table 4 Clinically significant improvement at five months in fatigue severity (checklist individual strength), functional impairment (SF-36), and school attendance (mean percentage) and self rated improvement by treatment group

\begin{tabular}{|c|c|c|c|}
\hline Condition & No (\%) improved & Treatment effect $(95 \% \mathrm{Cl})$ & $P$ value \\
\hline \multicolumn{4}{|l|}{ Fatigue severity* } \\
\hline Cognitive behaviour & $21 / 35(60)$ & 0.39 (0.17 to 0.61$)$ & 0.01 \\
\hline Waiting list & $7 / 34 \quad(21)$ & & \\
\hline \multicolumn{4}{|l|}{ Physical functioning $\dagger$} \\
\hline Cognitive behaviour & $22 / 35(63)$ & 0.39 (0.17 to 0.61$)$ & 0.01 \\
\hline Waiting list & $8 / 34 \quad(24)$ & & \\
\hline \multicolumn{4}{|c|}{ Full school attendance } \\
\hline Cognitive behaviour & $19 / 33(58)$ & 0.29 (0.06 to 0.53$)$ & 0.02 \\
\hline Waiting list & $10 / 34(29)$ & & \\
\hline \multicolumn{4}{|c|}{ Self rated improvement§ } \\
\hline Cognitive behaviour & $25 / 35(71)$ & 0.27 (0.04 to 0.78$)$ & 0.02 \\
\hline Waiting list & $15 / 34 \quad(44)$ & & \\
\hline
\end{tabular}

* Reliable change index $>1.96$ and cut off score of $\geq 35.7$.

tIncrease of $\geq 50$ or end score of $\geq 75$.

士Two participants were left out of analyses because they had taken their final examinations and therefore were not required to attend school at five months.

$\S$ Answer "yes" to statement "I have completely recovered" or "I feel much better but still experience some symptoms."
The prevalence of additional symptoms decreased significantly in immediate treatment group. Nevertheless, as in a previous report, ${ }^{21}$ many young people in both groups continued to report additional symptoms. Apparently, a complete resolution of additional symptoms is not a requirement of recovery, as has been suggested before. ${ }^{22}$

This study is the first randomised controlled trial to show that cognitive behaviour therapy can successfully be used to treat adolescents with chronic fatigue syndrome. As the prevalence of chronic fatigue syndrome seems to be lower than previously thought, we would not recommend widespread implementation of cognitive behaviour therapy but suggest that treatment should be centralised in specialised medical centres so that therapists can accumulate knowledge and maintain proficiency.

We thank all participants and their parents, Lida Nabuurs for assisting in data collection; and Maaike van Kuijk, Esther Meijer, and Thea Berends for carrying out the therapy; and Judith Prins for her contribution to the study design.

Contributors: MS undertook the allocation procedure, coordinated data collection from March 2002, performed all analyses, and wrote the paper. LWAMdJ contributed to the design of the study, treated some of the participants, screened all patients, and contributed to the writing. TJWF was responsible for diagnoses and referral to department of child psychology and contributed to editing the paper. SWBH contributed to the design of the study, undertook the allocation procedure, and coordinated data collection until March 2002. GB developed the original idea, was responsible for study coordination, training, and supervision of therapists, and contributed to editing of the paper. GB is the guarantor.

Funding: Foundation for Children's Welfare Stamps Netherlands (Stichting Kinderpostzegels Nederland) and the ME Society (ME Stichting).

Competing interests: None declared.

Ethical approval: Human Ethics Committee of the University Medical Centre Nijmegen. 
Table 5 Treatment effects on fatigue severity (checklist individual strength), functional impairment (SF-36), and school attendance (mean percentage) by activity pattern in patients assigned to cognitive behaviour therapy

\begin{tabular}{|c|c|c|c|c|c|}
\hline \multirow[b]{2}{*}{ Activity* pattern } & \multicolumn{2}{|c|}{ Mean score (SD) } & \multirow[b]{2}{*}{ Mean difference within groups $(95 \% \mathrm{Cl})$} & \multirow[b]{2}{*}{ Mean difference between groups ( $95 \% \mathrm{Cl}$ ) } & \multirow[b]{2}{*}{$P$ value } \\
\hline & 0 months & 5 months & & & \\
\hline \multicolumn{6}{|l|}{ Fatigue severity } \\
\hline Passive & $52.4(4.2)$ & $35.0(19.0)$ & $-17.4(-30.1$ to -4.7$)$ & $8.9(-3.3$ to 21.1$)$ & 0.147 \\
\hline Active & $52.6(3.9)$ & $26.3(14.8)$ & $-26.3(-32.8$ to -19.8$)$ & & \\
\hline \multicolumn{6}{|c|}{ Physical functioning } \\
\hline Passive & $37.0(17.8)$ & $59.0(35.7)$ & $22.0(4.7$ to 39.3$)$ & $10.0(-3.3$ to 21.1$)$ & 0.336 \\
\hline Active & $43.5(14.8)$ & $75.4(23.0)$ & 32.0 (20 to 43.4) & & \\
\hline \multicolumn{6}{|l|}{ School attendance } \\
\hline Passive & $21.0(35.2)$ & $54.1(44.6)$ & 33.1 (5.8 to 60.4$)$ & $4.1(-29.0$ to 37.3$)$ & 0.801 \\
\hline Active & $59.7(35.2)$ & $88.7(26.1)$ & 29.0 (10.2 to 47.8$)$ & & \\
\hline
\end{tabular}

*Passive $n=10$; active $n=23$

1 Fukuda K, Straus SE, Hickie I, Sharpe MC, Dobbins JG, Komaroff A, et al. The chronic fatigue syndrome: a comprehensive approach to its definition and study. Ann Intern Med 1994;121:953-9.

2 Reeves WC, Lloyd A, Vernon SD, Klimas N, Jason LA, Bleijenberg G, et al. Identification of ambiguities in the 1994 chronic fatigue syndrome research case definition and recommendations for resolution. BMC Health Serv Res 2003;3:25-33.

3 Chalder T, Goodman, R, Wessely, S, Hotopf M, Meltzer H. Epidemiology of chronic fatigue syndrome and self reported myalgic encephalomyelitis in 5-15 year olds: cross sectional study. BMJ 2003;327:654-5.

4 Prins JB, Bleijenberg G, Bazelmans E, Elving LD, de Boo Th, Severens JL, et al. Cognitive behaviour therapy for chronic fatigue syndrome: a multicentre randomised controlled trial. Lancet 2001;357:841-7.

5 Whiting P, Bagnall, A, Sowden AJ, Cornell JE, Mulrow CD, Ramirez G. Interventions for the treatment and management of CFS. JAMA 2001;286:1360-8.

6 Chalder T, Tong J, Deary V. Family cognitive behaviour therapy for chronic fatigue syndrome: an uncontrolled study. Arch Dis Child 2002;86:95-7.

7 Garralda ME, Rangel L. Annotation: chronic fatigue syndrome in children and adolescents. J Child Psychol Psychiatry 2002;43:169-76.

8 Carter BD, Edwards JF, Kronenber. WG, Michalczyk L Marshall GS. Case control study of chronic fatigue in pediatric patients. Pediatrics 1995;95:179-86.

9 Fritz U, McQuaid EL. Chronic medical conditions. Impact on development. In Sameroff AJ, Lewis M, Miller SM, eds. Handbook of developmental psychopathology. New York: Kluwer, 2000.

10 Van der Werf SP, Prins JB, Vercoulen JHMM, van der Meer JWM, Bleijenberg G. Identifying physical activity patterns in chronic fatigue syndrome using actigraphic assessment. J Psychosom Res 2000;49:372-9.

11 Bleijenberg G, Prins J, Bazelmans E. Cognitive-behavioral therapies. In: Jason LA, Fennel PA, Taylor RR, eds. Handbook of chronic fatigue syndrome. New Jersey: John Wiley, 2003.

12 Vercoulen JHHM, Swanink CMA, Galama JMD, Fennis JFM, van der Meer JWM, Bleijenberg G. Dimensional assessment in chronic fatigue syndrome. I Psychosom Res 1994;38:383-92.

\section{What is already known on this topic}

Cognitive behaviour therapy is an effective treatment for chronic fatigue syndrome in adults, and one uncontrolled study has shown that it can reduce fatigue in adolescents

Chronic fatigue syndrome in adolescents can affect normal development

\section{What this study adds}

A cognitive behaviour therapy programme based on gradually increasing activity and challenging perpetuating beliefs helped adolescents with chronic fatigue syndrome

Relatively active patients as well as those with a passive physical activity pattern benefited from tailored therapy
13 Beurskens AJHM, Bültmann U, Kant IJ, Vercoulen JHMM, Bleijenberg G, Swaen GMH Fatigue amongst working people: validity of a questionnaire. Occup Environm Med 2000;57:353-7.

14 Steward AL, Hays RD, Ware JE Jr. The MOS short form general health survey: reliability and validity in a patient population. Med Care 1998;26:724-35.

15 Bell DS, Jordan K, Robinson M. Thirteen-year follow-up of children and adolescents with chronic fatigue syndrome. Pediatrics 2001;107:994-8

16 Vercoulen JHHM, Swanink CMA, Zitman FG, Vreden SGS, Hoofs MPE, Fennis JFM, et al. A randomised, double-blind, placebo controlled study of fluoxentine in chronic fatigue syndrome. Lancet 1996;347:858-61.

17 Vercoulen JHHM, Bazelmans E, Swanink CMA, Fennis JFM, Galama JMD, Jongen PJH et al. Physical activity in the chronic fatigue syndrome: assessment and its role in fatigue. J Psychiatr Res 1997;31:661-73.

18 Faul F, Erdfelder E, Gpower: a priori, post-hoc, and compromise power analyses for MS-DOS [computer program]. Bonn, FRG: Bonn University, Department of Psychol1992.

19 Jacobsen NS, Truax P. Clinical significance: a statistical approach to defining meaningful change in psychotherapy. J Cons Clin Psychol 1991;59:12-9.

20 Powell P, Bentall R, Nye F, Edwards RH. Randomised controlled trial of patient education to encourage graded exercise in chronic fatigue syndrome. BMJ 2001;322:387-90.

21 Bell DS, Jordan K, Robinson M. Thirteen-year follow-up of children and adolescent with chronic fatigue syndrome. Pediatrics 2001;107: 994-8.

22 Gill AC, Dosen A, Ziegler JB. Chronic fatigue in adolescents: a follow-up study. Arch Pediatr Adolesc Med 2004;158:225-9.

(Accepted 7 October 2004)

doi 10.1136/bmj.38301.587106.63

Expert Centre Chronic Fatigue, University Medical Centre Nijmegen, PO Box 9101, $6500 \mathrm{HB}$, Netherlands

Maja Stulemeijer junior researcher

Sigrid W B Hoogveld junior researcher

Gijs Bleijenberg professor of psychology

Department of Medical Psychology, University Medical Centre Nijmegen Lieke W A M de Jong child psychologist

Department of Paediatrics, University Medical Centre Nijmegen

Theo J W Fiselier paediatrician

Correspondence to: G Bleijenberg G.Bleijenberg@nkcv.umcn.nl

\section{Amendment}

The numbers in the abstract have been altered for clarity. Under participants, the total number randomised (71) has been given, rather than the number after exclusions (69). In the results section, the number 58 has been corrected to 62 . 